\title{
A New Method for Detection of Active Brain Regions with Event-Related fMRI Time Series Analysis
}

\author{
Juliano J. Duque, Marcio J. Sturzbecher, Draulio B. Araújo, Joaquim C. Felipe \\ Department of Physics and Mathematics \\ Faculty of Philosophy, Sciences and Letter of Ribeirão Preto \\ University of São Paulo, Brazil \\ julianojd@gmail.com,marcio@biomag.usp.br,draulio@biomag.usp.br,jfelipe@ffclrp.usp.br
}

\begin{abstract}
Functional magnetic resonance imaging (fMRI) has been applied with several purposes in clinical environment as well as in basic neuroscience studies.

The main purpose of fMRI is to map brain activity, which is achieved by applying statistical methods to time series of each voxel intensity, related to changes in blood oxygen level, in response to a short stimulus.

In this work, we propose a new method for the analysis of event-related fMRI based in the use of the Dynamic Time Warping technique to compare pixel intensity time series. The results show the ability to discriminate activated brain regions, when subjects were engaged in a motor task.
\end{abstract}

\section{Introduction}

Functional neuroimaging is rapidly evolving in recent years. Among many techniques capable of characterizing brain activity, functional Magnetic Resonance Imaging (fMRI) deserves special attention. It allows the non-invasive mapping of the human brain with good spatiotemporal resolution [1], which opens the perspective of applications from basic neuroscience to clinical studies. For instance, it has been useful in pre-surgical procedures, by determining eloquent cortical areas which should be kept intact during surgical procedure. This mapping contributes essentially in the evaluation, planning and secure issues related to the surgical action. Moreover, it opens a window into the human brain, unveiling certain aspects of its functional patterns.

fMRI was first proposed in 1991 by Belliveau and colleagues [2], who demonstrated the possibility of mapping the primary visual cortex, by detecting contrast changes on magnetic resonance images (MRI). By then, it was used of an intravascular paramagnetic contrast agent. One year later, Kenneth Kwong [3] and Seiji Ogawa [4] proposed another contrast method, based on an endogenous mechanism, known as BOLD (Blood Oxygen Level Dependent) contrast. This method is based on the fact that deoxi-hemoglobin $(\mathrm{dHb})$ and oxi-hemoglobin $(\mathrm{OHb})$ have distinct magnetic behavior: first is paramagnetic while the second is diamagnetic. During brain activity, there is a local increase of blood flow, which is not followed by the same amount of oxygen consumption. Therefore, there is a decrease in the local concentration of $\mathrm{dHb}$, changing, in its turn, image contrast. Unfortunately, such changes are not high (on the order of $3 \%$ ), precluding direct visual inspection and demanding the use of computational algorithms to identify areas that had their contrast modulated [5].

In order to study brain activation with fMRI, a series of stimuli are presented to produce specific neuronal responses, which are intercalated with baseline periods. In the so-called event-related (ERfMRI) paradigm, a series of short stimulus are followed by a relatively long period of baseline. Brain regions that responded to the stimulus will have a hemodynamic response function (HRF) similar to the one shown in Figure 1. This paradigm presents several advantages, among the most important of which are (1) better temporal resolution; (2) the possibility of mixing different cognitive processes; (3) the possibility of dissociating cortical areas involved in different tasks; and (4) smaller contamination of cognitive artifacts.

The analysis of fMRI time series is designed to identify voxels in which brightness was modulated by the task. One possibility is to compute a Student-t test, which statistically quantifies differences between averages of two signals, from images of active and baseline states. Although possible, it is highly susceptible to noise, once short changes in the resonance signal can produce spurious points in the resultant images. Thus, finding an efficient statistical 
technique to treat $\mathrm{AMRI}$ time series has gained much attention [6] [7]. In applications of fMRI, such as helping pre-surgical mapping and analysis of brain regions affected by some disease, it is essential to ensure that the applied method will not produce false positive finds.

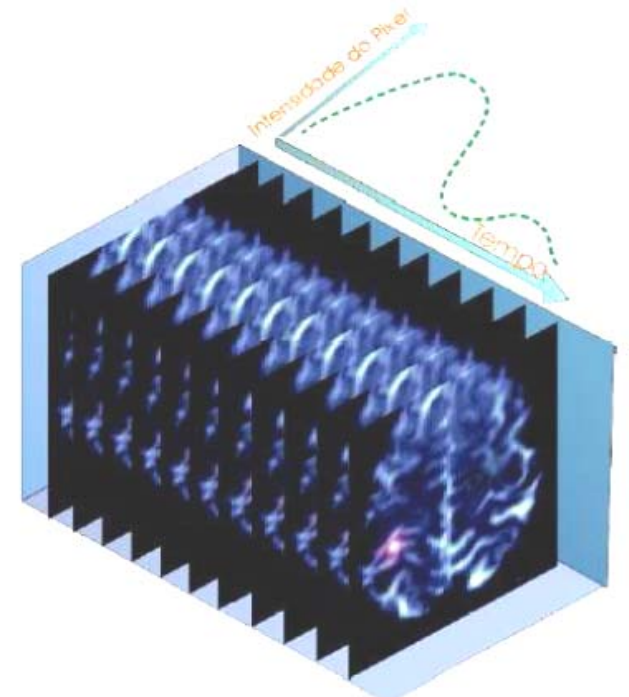

Figure 1. Hemodynamic response function

Specifically concerning ER-fMRI there are some usual statistical analysis methods, being the simplest one a convolution of a gamma variate function with a modeled standard HRF [8]. Such approach produces statistical maps capable of identifying regions, which HRFs are similar to the reference function [9]. However, to use this technique, one needs to model a standard HRF, which is not trivial [10].

In the same frame of research, we herein propose a new deterministic method for analyzing fMRI time series, acquired during event-related experiments. This method consists of the comparison between two time series: the one of each pixel and a reference function. The result is a measure of the similarity between the two series [11]. Therefore, by plotting the similarity value obtained for each pixel from a brain slice image, it is possible to identify the brain regions that were active according to the applied stimuli. The individual evaluation of each pixel time series, comparing it directly against the stimuli behavior function with a suitable cut-value, assures that no false positive point or region will be generated due to the analysis method.

\section{Dynamic Time Warping}

Dynamic Programming [12] is a numeric technique for comparing vectors, consisting in the evaluation of all possible combinations of distance results between pairs of correspondent adjacent attributes, aiming at minimizing a parameter that represents some kind of "cost" function.

The Dynamic Time Warping [13] [14] is an efficient technique to compare time series, based on the application of Dynamic Programming. Its main goal is to keep close series that have similar behavior, but are delayed or distort along the time axis. Thus, this technique has a good sensibility to warp. It comes from the fact that comparisons between corresponding points are not rigid, allowing that points of a series can be compared with adjacent ones in other series, as illustrated in Figure 2.

Suppose we have two series, $\mathrm{Q}=(\mathrm{q} 1, \mathrm{q} 2, \ldots, \mathrm{qn})$ and $\mathrm{C}=(\mathrm{c} 1, \mathrm{c} 2, \ldots, \mathrm{cm})$, with respective sizes $n$ and $m$. In order to compare them using Dynamic Time Warping, a $n \times m$ matrix is built, whose $(\mathrm{i}, \mathrm{j})$ element contains the distance d(qi, cj). Typically, the Euclidian distance is used. Each element of the matrix corresponds to the distance between the points that it represents.
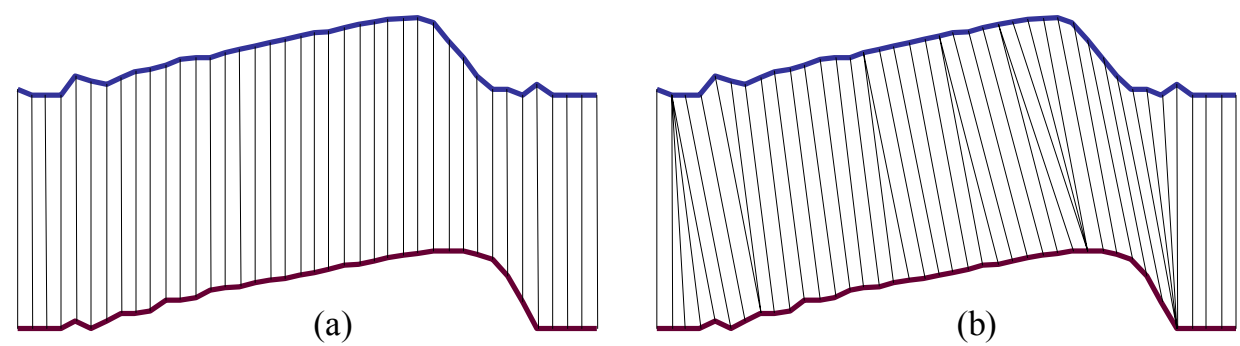

Figure 2. Comparison between series: a) conventional; b) with Dynamic Time Warping 
An adjustment route $\mathrm{W}=(\mathrm{w} 1, \mathrm{w} 2, \ldots, \mathrm{wk})$ consists of a set of contiguous matrix elements that define a mapping between $\mathrm{Q}$ and $\mathrm{C}$. The adjustment route is defined by the following rules [13]:

- It starts at cell $(1,1)$ and finishes at cell $(n, m)$;

- The sequence of the route must have adjacent elements of the matrix (including diagonally);

- The sequence must not go back in the route.

There are several possible adjustment routes. However, the best route is the one that minimizes the deformation cost, in other words, the route whose accumulated distance is minimal. This minimal accumulated distance is defined as the Warp Distance, shown in equation (1).

$$
W D(Q, C)=\min \left(\sum_{k=1}^{K} w_{k} / K\right)
$$

Where $w_{k}$ is the $\mathrm{k}^{\text {th }}$ element of the adjustment route and $K$ is the number of elements of the adjustment route.

Warp Distance has succeeded in applications such as data mining, gesture recognition, robotics, speech processing and image processing [15].

\section{Proposed Method}

In this work we propose a method to evaluate the similarity between time series derived from variation on pixel intensity of brain fMRI images, due to stimulus applied under an event-related paradigm. The method consists of four main steps, presented in the following sections.

\subsection{Pre-processing of Time Series}

The original fMRI time series consists of a badly behaved signal, as shown in Figure 3. Before the similarity comparison with the reference function, the series will be pre-processed as follows:

- Noise reduction: smooth the curve, making a cleaner signal, to be compared with the reference gamma function (see Section 3.2);

- Offset translation: medial axis of the curve is translated to superpose the horizontal axis, in order to be compatible with the reference gamma function;

- Amplitude scaling: signal amplitude is modified to adapt it to reach a proportion between the axes similar to that presented by the reference function.

\subsection{Reference Function Determination}

The reference function of an impulse stimulus is generally modeled by a gamma variate function [10]. Its inference will be based on ten time series from representative brain regions, known a priori being active for the specific stimulus. This set of time series is then pre-processed, according to the description in Section 3.1. Subsequently, each series is sliced in the number of sequences of applied stimulus (epoch) and each epoch is divided in the number of time points. The most frequent value of each time point, considering all periods of the chosen pixels, is then identified to be one time reference point, composing a function that will be used as parameter to determine the gamma reference function period. The repetition of this period by the number of sequences will then compose the reference function.

\subsection{Application of Dynamic Time Warping}

The third step consists in applying Dynamic Time Warping, comparing the time series of each pixel with the reference function. For each time series, a distance matrix is built, and in each matrix the minimal cost path is identified, leading to the computation of the minimal accumulated distance, as described in Section 2. This final distance is the value that will be used to plot each pixel position with a different color, representing the similarity of its time series with the reference function.

\subsection{Pixel Activation Mapping}

The final step consists in constructing a color map for each acquisition image slice. The colors in the map represent the distance between the respective pixel to the reference function. According to the color of the pixels, it will be possible to identify the active brain regions.

\section{Experiments and Results}

Images were generated using EPI-BOLD-like sequences. In this experiment, a volunteer was asked to move his fingers of both hands. All images were obtained in a scanner of 1.5 T (Siemens, Magneton Vision).

Stimuli were performed for $3 \mathrm{sec}$, followed by a rest state of 20 sec. Meanwhile, 3 axial slices (128 x 128 pixels), positioned over the primary motor cortex were continuously acquired (3 images acquired during the activity state and 11 during the rest state) summing up a set of 14 images, for a complete stimulus-rest series, 
called epoch. The complete experiment was made by a total of 24 epochs, for each axial slice.

Each time series were derived from each one of the $128 \times 128$ pixels, for each slice, consisting of 336 time points (24 epochs, each one with 14 time points). Figure 3 shows two samples of series obtained.
The method described in Section 3 was applied on this result set of time series. After the pre-processing, the series shown in Figure 3 were modified as shown in Figure 4.

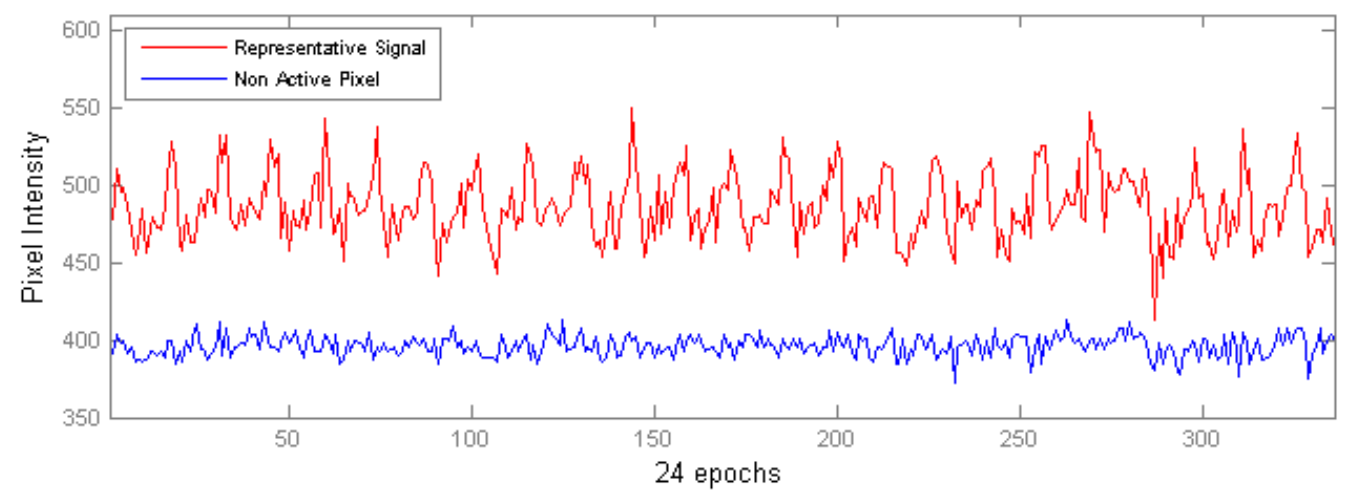

Figure 3. Two times series obtained: from an active pixel (red) and from an inactive pixel (blue)
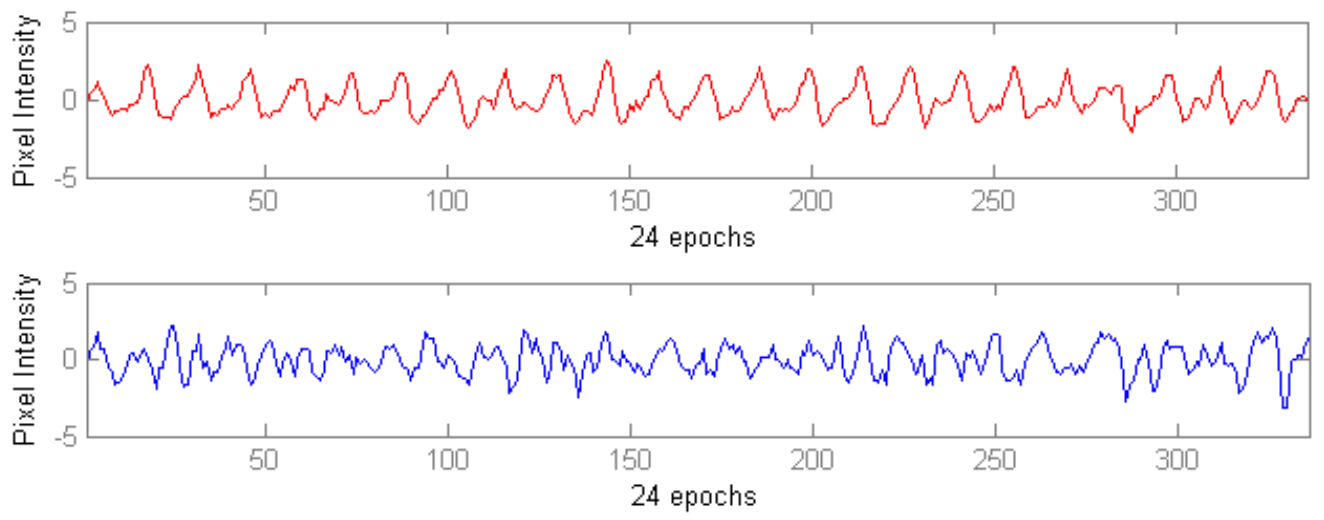

Figure 4. Time series modified after pre-processing: active pixel (red) and inactive pixel (blue)

The determination of the reference function was made from 10 pixels chosen from each of the 3 slices. Each time series was sliced in 24 epochs and from each epoch, 14 time points were identified. Then, the most frequent intensities for each time point were identified and the gamma function was parameterized according to these points. The resulting reference function is shown in Figure 5.

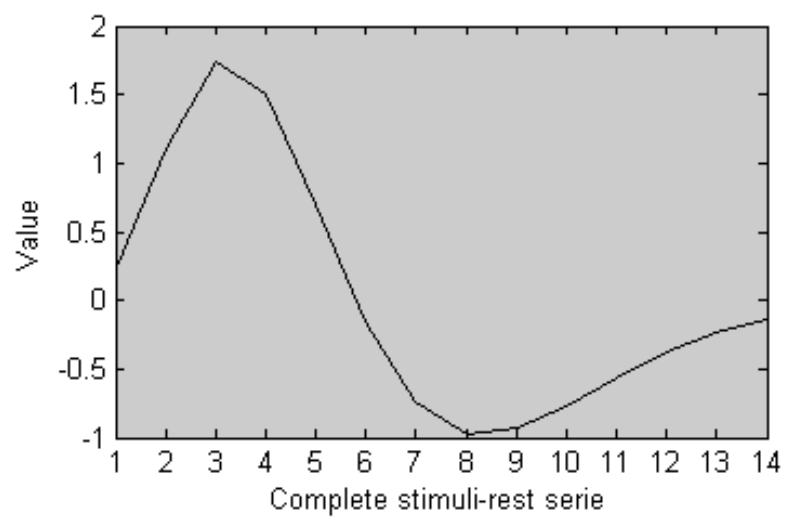

Figure 5. Resulting epoch of reference function 
Then, every pixel time series was compared with the reference function by the Dynamic Time Warping application, generating a matrix for each slice, containing a distance value for each pixel. Finally, this matrix was plotted, generating the color pixel maps, and superposed to the original slice image. Figure 6 shows the resulting maps for those 3 slices. Dark red color points represent the areas of highest activity, corresponding to the motor cortex. While the activity
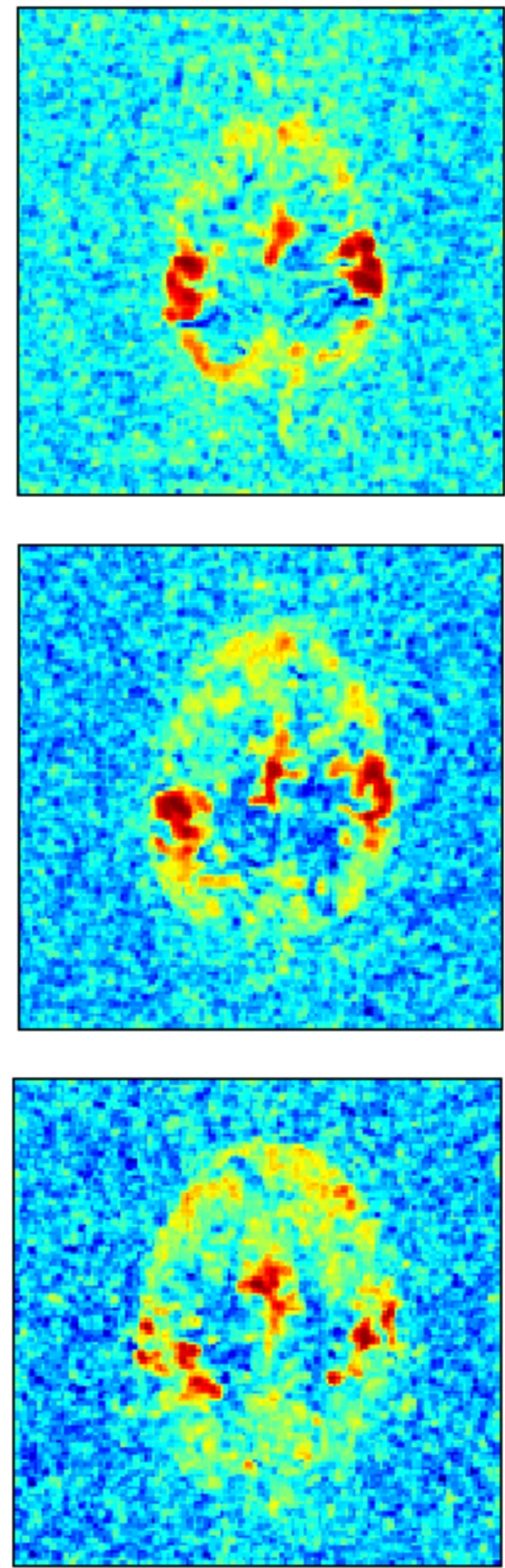

Figure 6. Color maps and superposed images for 3 slices level decreases, the color gradually changes from dark red to dark blue. In the superposed images, only pixels with warp distance lower than a cut value of 0.14 were plotted, detaching the activity areas.

The image time series analysis was implemented in Matlab (Mathworks, Sherborn, MA) software and the Dynamic Time Warping application was implemented in $\mathrm{C}++$ Builder (Borland, CA) environment.
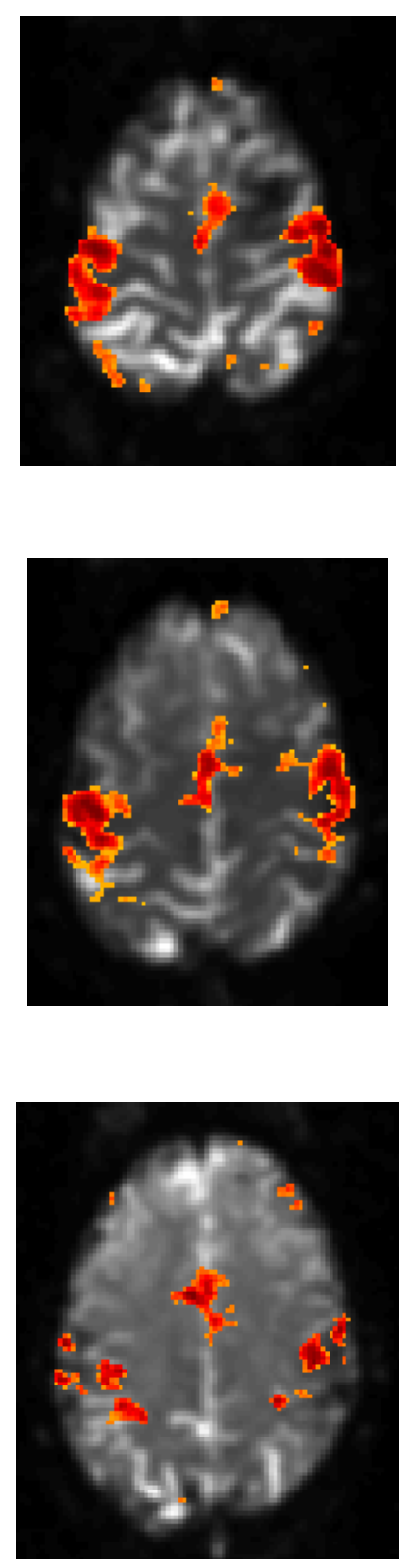


\section{Discussion and Conclusion}

The reference function, in Figure 5, reproduces the time course of the magnetic resonance signal, during a single trial experiment after a brief period of motor activation, in a representative pixel. As described in [10], the hemodynamic response peak is followed by an undershoot returning then to the baseline. In Figure 4 , this behavior can be clearly noticed in each epoch of the active pixel.

The resulting maps, shown in Figure 6, present coherence with an a priori known motor activation, in the primary motor cortex, as well as supplementary motor areas. These results are also consistent with results of other experiments concerning motor stimuli [10].

The results from this study give support to the hypothesis that event-related fMRI experiments may be consistently analyzed by the method proposed herein.

Considering that several applications of fMRI demand very high level of certainty about the results, the proposed method assures that no false positive find will be created, once the time series functions are directly compared to the reference function, and only the series that present a feasible adjustment to the reference function can be considered as representing active brain areas.

\section{Acknowledgment}

This work is partially supported by brazilian Fundação para o Amparo à Pesquisa no Estado de São Paulo (FAPESP).

\section{References}

[1] Dale, A.M., "Optimal Experimental Design for EventRelated fMRI", Human Brain Mapping, vol. 8, 1999, pp. 109-114.

[2] Belliveau, J.W., Kennedy, D.N., Mckinstry, R.C., Buchbinder, B.R., Weisskoff, R.M., Cohen, M.S., Vevea, J.M., Brady, T.J., and Rosen, B.R., "Functional mapping of the human visual cortex by magnetic resonance imaging", Science, vol. 254, 1991, pp. 716-719.

[3] Kwong, K.K., Beliveau, J.W., Chesler, D.A., Goldberg, I.E., Weisskoff, R.M., Poncelet, B.P., Kennedy, D.N., Hoppel, B.E., Cohen, M.S., Turner, R., Cheng, H., Brady, T.J., and Rosen, B.R., "Dynamic magnetic resonance imaging of human brain activity during primary sensory stimulation", Proc. of the National Academy of Science, vol. 89, 1992, pp. 5675-5679.
[4] Ogawa, S., Tank, D.W., Menon, R., Ellermann, J.M., Kim, S.G., Merkle, H., and Ugurbil, K., "Intrinsic signal changes accompanying sensory stimulation: functional brain mapping with magnetic resonance imaging", Proc. of the National Academy of Science, vol. 89, 1992, pp. 5951-5955.

[5] de Araujo, D. B., Sobre Neuroimagens Funcionais por Magnetoencefalografia e Ressonância Magnética: Novos Métodos e Aplicações, Tese de Doutorado, Departamento de Física e Matemática - FFCLRP, Universidade de São Paulo, Ribeirão Preto, Brasil, 2002.

[6] de Araujo, D. B., Salles, A., Tedeschi, W., Araujo, D., Santos, A. C., Baryshnikov, B., Wakai, R. T., and Baffa, O., "Spatiotemporal Patterns of Human Navigation Investigated by MEG and AMRI". Proceedings of the 13th International Conference on Biomagnetism, 2002, pp. 863-865.

[7] Araujo, D., de Araujo, D. B. ou Araujo, D. B., Pontes Neto, O. M., Velasco, T., Sakamoto, A., Simão, G. N., Ana, L. W., Leite, J. P., and Santos, A. C., "Language and Motor fMRI Activation in Polymicrogyric Cortex". Epilepsia, vol. 47, 2006, pp. 589-592.

[8] Clare, S., Functional MRI: methods and applications, Phd thesis. John Radcliffe Hospital. University of Oxford, Oxford, 1998.

[9] Friston, K. J., Holmes, A. P., Poline, J.B., Grasby, P. J., Williams, S. C. R., Frackowiak, R. S. J. and Turner, R., "Analysis of fMRI time-series revisited". Neuroimage, vol. 2, 1995, pp. 45-53.

[10] de Araújo, D.B., Tedeschi, W., Santos, A.C., Elias Jr., J., Neves, U.P.C., and Baffa, O., "Shannon entropy applied to the analysis of event-related fMRI time series", NeuroImage, vol.20, 2003, pp. 311-317.

[11] Keogh, E., A Tutorial on Indexing and Mining Time Series Data. Tutorial presented in IEEE International Conference on Data Mining. San Jose, USA, 2001.

[12] Gusfield, D., Algorithms on Strings, Trees and Sequences, Cambridge University Press, New York, NY, USA, 1999.

[13] Keogh, E., "Exact Indexing of Dynamic Time Warping", Proc. of the 28th Conference on Very Large Databases, Hong Kong, China, 2002, pp. 406-417.

[14] Keogh, E., and Pazzani, M. J., "Derivative Dynamic Time Warping", Proc. of the First SIAM International Conference on Data Mining, Chicago, USA, 2001.

[15] Felipe, J. C., Desenvolvimento de métodos para extração, comparação e análise de características intrínsecas de imagens médicas, visando à recuperação perceptual por conteúdo, Phd thesis, Institute of Mathematics and Computer Sciences - ICMC, University of São Paulo, São Carlos, Brazil, 2005. 